NTERnational Electronic Journal of Algebra

VOLUME 27 (2020) 178-193

DOI: $10.24330 /$ ieja. 663060

\title{
CHARACTERIZATIONS OF SOME CLASSES OF RINGS VIA LOCALLY SUPPLEMENTED MODULES
}

\author{
Farid Kourki and Rachid Tribak \\ Received: 24 March 2019; Accepted: 7 October 2019 \\ Communicated by Nanqing Ding
}

\begin{abstract}
We introduce the notion of locally supplemented modules (i.e., modules for which every finitely generated submodule is supplemented). We show that a module $M$ is locally supplemented if and only if $M$ is a sum of local submodules. We characterize several classes of rings in terms of locally supplemented modules. Among others, we prove that a ring $R$ is a Camillo ring if and only if every finitely embedded $R$-module is locally supplemented. It is also shown that a ring $R$ is a Gelfand ring if and only if every $R$-module having a finite Goldie dimension is locally supplemented.
\end{abstract}

Mathematics Subject Classification (2010): 13C13, 13C99, 16D10

Keywords: Camillo ring, Gelfand ring, locally supplemented module, semiperfect ring, supplemented module

\section{Introduction}

Throughout this article, all rings are commutative with identity and all modules are unital. Let $R$ be a ring. We denote respectively by $\operatorname{Spec}(R)$ and $\operatorname{Max}(R)$ the set of all prime ideals of $R$ and the set of all maximal ideals of $R$. Let $M$ be an $R$-module and let $x \in M$. We denote the annihilator of $M$ (of $x$ ) by $A n n(M)$ (by $\operatorname{Ann}(x)$ ), i.e. $\operatorname{Ann}(M)=\{r \in R \mid r m=0$ for every $m \in M\}$ and $A n n(x)=\{r \in R \mid r x=0\}$. We use $\operatorname{Rad}(M), \operatorname{Soc}(M), E(M)$, and $\operatorname{End}_{R}(M)$ to denote the radical, the socle, the injective hull of $M$, and the endomorphism ring of $M$, respectively. The notation $N \subseteq M$ means that $N$ is a subset of $M$ and the notation $N \leq M$ means that $N$ is a submodule of $M$. We say that a submodule $N$ of $M$ is a small submodule and we write $N \ll M$ if $M \neq N+L$ for any proper submodule $L$ of $M$. A nonzero module $M$ is called hollow if every proper submodule is small in $M$. Let $N$ be a submodule of a module $M$. A submodule $K$ of $M$ is called a supplement of $N$ in $M$ if $K$ is minimal with respect to the property $M=N+K$, equivalently, $M=N+K$ and $N \cap K \ll K$. If every submodule of $M$ has a supplement in $M$, then $M$ is called supplemented. The module $M$ will be called a local module if $\operatorname{Rad}(M)$ is a small maximal submodule of $M$. 
In Section 2, we introduce the notion of locally supplemented modules. A module $M$ is said to be locally supplemented if every finitely generated submodule of $M$ is supplemented. We investigate some basic properties of locally supplemented modules and we establish a characterization of this type of modules. It is shown that a module $M$ is locally supplemented if and only if $M$ is a sum of local submodules of $M$.

The results obtained in Section 3 can be considered as the principal motivation behind this work. In fact, a number of classes of rings are characterized in terms of locally supplemented modules. The class of rings $R$ for which every finitely embedded module is locally supplemented, is shown to be exactly that of Camillo rings (that is, the rings $R$ for which $\operatorname{Hom}_{R}\left(E\left(R / \mathfrak{m}_{1}\right), E\left(R / \mathfrak{m}_{2}\right)\right)=0$ for any two distinct maximal ideals $\mathfrak{m}_{1}$ and $\mathfrak{m}_{2}$ of $R$ ). Also, we characterize the class of rings $R$ for which every $R$-module having a finite Goldie dimension is locally supplemented as that of the Gelfand rings (i.e., the rings in which every prime ideal is contained in only one maximal ideal).

\section{Some properties of locally supplemented modules}

Definition 2.1. An $R$-module $M$ is called locally supplemented if every finitely generated submodule of $M$ is supplemented.

Example 2.2. (i) It is clear that every artinian module is locally supplemented.

(ii) From [14, Propositions A.7 and A.8], it follows that a finitely generated $\mathbb{Z}$ module is supplemented if and only if it is a torsion module. This implies that locally supplemented $\mathbb{Z}$-modules are exactly the torsion $\mathbb{Z}$-modules.

It is of natural interest to compare the class of locally supplemented modules and that of supplemented modules. We begin by giving some locally supplemented modules which are not supplemented.

Example 2.3. (i) Let $R$ be a semiperfect ring which is not perfect (for example we can take a discrete valuation ring $R$ ). Then every $R$-module is locally supplemented by [14, Theorem 4.41]. Consider the $R$-module $M=R^{(\mathbb{N})}$. So $M$ is locally supplemented. However, $M$ is not supplemented by [5, Theorem 1].

(ii) It is well known that every artinian module is supplemented. Let $p$ be a prime integer and let $M=\oplus_{i=1}^{\infty} M_{i}$ with $M_{i} \cong \mathbb{Z}\left(p^{\infty}\right)$ for all $i \geq 1$. Let $N$ be a finitely generated submodule of $M$. Then $N \subseteq \oplus_{j=1}^{n} M_{i_{j}}$ for some positive integers $i_{1}, \ldots, i_{n}$. It follows that $N$ is artinian and so supplemented. Hence $M$ is locally supplemented. On the other hand, $M$ is not supplemented by [14, Propositions A.7 and A.8]. 
In [20, p. 45 Remark], Zöschinger exhibits a supplemented $R$-module $M$ over a noncommutative ring $R$ such that $M$ has a cyclic submodule which is not supplemented. In particular, the module $M$ is not locally supplemented. One may ask whether any supplemented module over a commutative ring is locally supplemented. But as far as we know this question is still unanswered. However, there exist some partial answers to this question.

Example 2.4. Any supplemented $R$-module over a commutative noetherian ring $R$ is locally supplemented by [21, Folgerung 2 p. 55].

Recall that a ring $R$ is called semiperfect if $R$ is semilocal and the idempotents lift modulo $\operatorname{Rad}(R)$. It is well known that a commutative ring $R$ is semiperfect if and only if $R$ is a finite direct product of local rings.

Lemma 2.5. (See [21, Satz 1.6 and Folgerung p. 52]) Let $M$ be a finitely generated $R$-module over a commutative ring $R$. Then the following are equivalent:

(i) $M$ is supplemented;

(ii) $M$ is locally supplemented;

(iii) $R / \operatorname{Ann}(x)$ is a semiperfect ring for all $0 \neq x \in M$;

(iv) $R / \operatorname{Ann}(M)$ is a semiperfect ring.

The next result is a direct consequence of Lemma 2.5.

Proposition 2.6. The following are equivalent for an $R$-module $M$ :

(i) $M$ is locally supplemented;

(ii) $R x$ is supplemented for every $x \in M$;

(iii) $R / A n n(x)$ is a semiperfect ring for every $0 \neq x \in M$;

(iv) $R / \operatorname{Ann}(N)$ is a semiperfect ring for every nonzero finitely generated submodule $N$ of $M$.

Recall that a ring $R$ is called a Gelfand ring if every prime ideal of $R$ is contained in only one maximal ideal of $R$. Using [21, Folgerung p. 50], it follows that a ring $R$ is semiperfect if and only if $R$ is semilocal and Gelfand. This fact has the following consequence:

Proposition 2.7. Let $R$ be a ring. If $I_{1}$ and $I_{2}$ are proper ideals of $R$ such that $R / I_{1}$ and $R / I_{2}$ are semiperfect rings, then $R / I_{1} I_{2}$ and $R / I_{1} \cap I_{2}$ are also semiperfect rings.

Let $M$ be an $R$-module. The support of $M$ is denoted by $\operatorname{Supp}(M)$ and it is defined by $\operatorname{Supp}(M)=\{\mathfrak{p} \in \operatorname{Spec}(R) \mid \mathfrak{p} \supseteq \operatorname{Ann}(N)$ for some cyclic submodule $N$ 
of $M\}$. Note that $\operatorname{Supp}(M)=\left\{\mathfrak{p} \in \operatorname{Spec}(R) \mid M_{\mathfrak{p}} \neq 0\right\}$. The set of all maximal ideals in $\operatorname{Supp}(M)$ will be denoted by $\operatorname{Max} \operatorname{Supp}(M)$. Then $\operatorname{Max} \operatorname{Supp}(M)=\{\mathfrak{m} \in$ $\left.\operatorname{Max}(R) \mid M_{\mathfrak{m}} \neq 0\right\}$.

Remark 2.8. (i) It is clear that any submodule of a locally supplemented module is again locally supplemented. Since every factor of a semiperfect ring is semiperfect, it follows that the class of locally supplemented modules is closed under factor modules.

(ii) Let $M$ be an $R$-module. From Propositions 2.6 and 2.7, we see that any sum of locally supplemented submodules of $M$ is locally supplemented. This implies that $M$ contains a unique maximal locally supplemented submodule which will be denoted by $K(M)$. By Proposition 2.6, we have $K(M)=\{x \in M \mid R x$ is supplemented $\}=\{x \in M \mid x=0$ or $R / \operatorname{Ann}(x)$ is a semiperfect ring $\}$. Let $\mathfrak{m}$ be a maximal ideal of $R$. As in [21, p. 53], let $K_{\mathfrak{m}}(M)=\{x \in M \mid x=0$ or the only maximal ideal over $\operatorname{Ann}(x)$ is $\mathfrak{m}\}$. By [21, Satz 2.3], we have $K(M)=$ $\bigoplus_{\mathfrak{m} \in \operatorname{MaxSupp}(M)} K_{\mathfrak{m}}(M)$.

Recall that an $R$-module $M$ is said to be local if $M$ contains a proper submodule which contains all other proper submodules. It is easy to see that a module $M$ is local if and only if it is hollow and cyclic (see [7, 2.15]).

If $M$ is an $R$-module and $P=\prod_{\mathfrak{m} \in \operatorname{MaxSupp}(M)} M_{\mathfrak{m}}$ is the direct product of the localizations $M_{\mathfrak{m}}$ 's, then we denote by $\Psi$ the map which sends an element $x \in M$ to the element in $P$ whose $M_{\mathfrak{m}}$-th coordinate in $M_{\mathfrak{m}}$ is $x / 1$.

The next result describes the structure of locally supplemented modules.

Theorem 2.9. The following are equivalent for an $R$-module $M$ :

(i) $M$ is locally supplemented;

(ii) $M=K(M)$;

(iii) $M=\bigoplus_{\mathfrak{m} \in \operatorname{MaxSupp}(M)} K_{\mathfrak{m}}(M)$;

(iv) $M=\sum_{\lambda \in \Lambda} L_{\lambda}$ where $L_{\lambda}$ is a local submodule of $M$ for all $\lambda \in \Lambda$;

(v) $\Psi$ is an isomorphism between $M$ and $\bigoplus_{\mathfrak{m} \in \operatorname{MaxSupp}(M)} M_{\mathfrak{m}}$.

(vi) For every $\mathfrak{m} \in \operatorname{Max} \operatorname{Supp}(M), K_{\mathfrak{m}}(M)$ is isomorphic to $M_{\mathfrak{m}}$ under the map $x \mapsto x / 1$.

Proof. (i) $\Leftrightarrow$ (ii) This is obvious.

(ii) $\Leftrightarrow$ (iii) This follows from [21, Satz 2.3].

(iii) $\Rightarrow$ (iv) This follows from the fact that for every nonzero cyclic submodule of $K_{\mathfrak{m}}(M)(\mathfrak{m} \in \operatorname{Max} \operatorname{Supp}(M))$ is a local module. 
(iv) $\Rightarrow$ (i) Let $N$ be a finitely generated submodule of $M=\sum_{\lambda \in \Lambda} L_{\lambda}$. So $N \leq L_{\lambda_{1}}+\cdots+L_{\lambda_{n}}$ for some $\lambda_{1}, \ldots, \lambda_{n} \in \Lambda$. Note that $L_{\lambda_{1}}+\cdots+L_{\lambda_{n}}$ is locally supplemented by $[7,20.20]$ and Lemma 2.5. Therefore $N$ is supplemented and consequently $M$ is locally supplemented.

(i) $\Leftrightarrow(\mathrm{v}) \Leftrightarrow(\mathrm{vi})$ By $[13$, Theorem B].

The next result is a direct consequence of Theorem 2.9.

Corollary 2.10. Let $M$ be an indecomposable R-module. Then $M$ is locally supplemented if and only if $M=K_{\mathfrak{m}}(M)$ for some maximal ideal $\mathfrak{m}$ of $R$.

The next result is taken from [3, Lemma 1.10].

Lemma 2.11. Let $M$ be an $R$-module and let $\mathfrak{p}$ be a prime ideal of $R$. Then $M_{\mathfrak{p}}=0$ if and only if $\operatorname{Hom}_{R}(M, E(R / \mathfrak{p}))=0$.

Applying Corollary 2.10 and Lemma 2.11, we obtain the following result.

Proposition 2.12. Let $R$ be a ring and let $\mathfrak{p} \in \operatorname{Spec}(R)$. Then the following conditions are equivalent:

(i) $E(R / \mathfrak{p})$ is a locally supplemented $R$-module;

(ii) $\operatorname{Max} \operatorname{Supp}(E(R / \mathfrak{p}))$ contains only one maximal ideal;

(iii) There exists a maximal ideal $\mathfrak{m}$ of $R$ such that $\operatorname{Hom}_{R}\left(E(R / \mathfrak{p}), E\left(R / \mathfrak{m}^{\prime}\right)\right)=$ 0 for any $\mathfrak{m}^{\prime} \in \operatorname{Max}(R) \backslash\{\mathfrak{m}\}$.

Combining Theorem 2.9 and $[7,20.18]$, we get the following corollary.

Corollary 2.13. Let $M$ be a locally supplemented module. If $N$ is a submodule of $M$ such that $M / N$ is finitely generated, then $N$ has a supplement in $M$.

The product of locally supplemented modules is not, in general, locally supplemented as shown below.

Example 2.14. Let $p$ be a prime integer and consider the family $\left\{M_{i}\right\}_{i \geq 1}$ of $\mathbb{Z}$ modules where $M_{i}=\mathbb{Z} / p^{i} \mathbb{Z}$ for all $i \geq 1$. Let $M=\prod_{i \geq 1} M_{i}$. It is clear that each $M_{i}(i \geq 1)$ is a locally supplemented $\mathbb{Z}$-module. On the other hand, taking $x=\left(x_{i}\right)_{i \geq 1} \in M$ with $x_{i}=1+p^{i} \mathbb{Z}$ for every $i \geq 1$, we have $A n n_{\mathbb{Z}}(x)=0$. So $x \notin K(M)$. Hence $M$ is not locally supplemented by Theorem 2.9 .

We conclude this section by presenting an example which shows that the class of locally supplemented modules is not closed under module extensions. 
Example 2.15. Let $K$ be a field and let the commutative ring $S=\prod_{n \in \mathbb{N}} K_{n}$, where $K_{n}=K$ for all $n \in \mathbb{N}$. Let $R$ be the subring of $S$ consisting of all sequences $\left\{d_{n}\right\}$ such that there exist $c \in K, k \in \mathbb{N}$ with $d_{n}=c$ for all $n \geq k$. Then $R$ is a von Neumann regular ring and $\operatorname{Soc}(R)$ consists of all sequences $\left\{d_{n}\right\}$ in $R$ such that $d_{n}=0$ for all $n \geq k$ for some $k \in \mathbb{N}$. Clearly, $\operatorname{Soc}(R)$ is a maximal ideal of $R$ since the ring $R / \operatorname{Soc}(R)$ is isomorphic to the field $K$. Consider the following short exact sequence

$$
0 \rightarrow \operatorname{Soc}(R) \rightarrow R \rightarrow R / \operatorname{Soc}(R) \rightarrow 0 .
$$

Note that $\operatorname{Soc}(R)$ and $R / \operatorname{Soc}(R)$ are locally supplemented $R$-modules. However, $R$ is not a locally supplemented $R$-module, since otherwise $R$ will be a semiperfect ring (see Proposition 2.6) and hence $R$ will be a semisimple ring.

\section{Rings whose modules are locally supplemented}

In this section, we characterize several important classes of rings in terms of locally supplemented modules. We begin with the following result which is a direct consequence of [14, Theorem 4.41 and Corollary 4.42].

Proposition 3.1. The following conditions are equivalent for a ring $R$ :

(i) Every R-module is locally supplemented;

(ii) Every injective R-module is locally supplemented;

(iii) The $R$-module $R$ is locally supplemented;

(iv) The $R$-module $R$ is supplemented;

(v) $R$ is semiperfect.

Recall that an integral domain $R$ is called $h$-local if the following two conditions are satisfied:

(1) Each nonzero ideal of $R$ is contained in only finitely many maximal ideals of $R$;

(2) Each nonzero prime ideal of $R$ is contained in only one maximal ideal of $R$.

Remark 3.2. Let $R$ be a domain which is not a field and let $K$ be its quotient field. Using [13, Corollary 4.7] and Theorem 2.9, we conclude that $R$ is an h-local domain if and only if $K / R$ is a locally supplemented $R$-module. In particular, if $R$ is a Dedekind domain, then the $R$-module $K / R$ is locally supplemented.

We call a ring $R$ almost semiperfect if $R / I$ is a semiperfect ring for any nonzero ideal $I$ of $R$. Clearly, every semiperfect ring is almost semiperfect. The following Lemma is an easy consequence of [21, Folgerung p. 50]. 
Lemma 3.3. Let $R$ be an integral domain. Then $R$ is almost semiperfect if and only if it is h-local.

An $R$-module $M$ is called unfaithful if $\operatorname{Ann}_{R}(M) \neq 0$.

Proposition 3.4. The following are equivalent for a ring $R$ :

(i) Every unfaithful $R$-module is locally supplemented;

(ii) $R$ is almost semiperfect;

(iii) $R$ is semiperfect or $R$ is an h-local domain.

Proof. (i) $\Rightarrow$ (ii) Let $I$ be a nonzero ideal of $R$. Since $A n n_{R}(R / I)=I \neq 0, R / I$ is an unfaithful $R$-module and so it is locally supplemented. But $R / I$ is a cyclic $R$-module. Then $R / I$ is a supplemented $R$-module. This implies that $R / I$ is a supplemented $R / I$-module. Hence $R / I$ is a semiperfect ring (see Proposition 3.1).

(ii) $\Rightarrow$ (iii) Assume that $R$ is not an h-local domain. So $R$ is not an integral domain by Lemma 3.3. It follows that $R$ contains two nonzero elements $a$ and $b$ such that $a b=0$. Since $R$ is almost semiperfect, $R / a R$ and $R / b R$ are semiperfect. Applying Proposition 2.7, it follows that $R / a R b R=R / a b R$ is semiperfect. Hence $R$ is a semiperfect ring.

(iii) $\Rightarrow$ (i) From Lemma 3.3, it follows that $R$ is almost semiperfect. Now let $M$ be an unfaithful $R$-module. Then $R / A n n(M)$ is a semiperfect ring. By Proposition $3.1, M$ is locally supplemented as an $R / \operatorname{Ann}(M)$-module and so also as an $R$ module.

A ring $R$ is called Camillo if $\operatorname{Hom}_{R}\left(E\left(R / \mathfrak{m}_{1}\right), E\left(R / \mathfrak{m}_{2}\right)\right)=0$ for any two distinct maximal ideals $\mathfrak{m}_{1}$ and $\mathfrak{m}_{2}$ of $R$. Sharpe and Vámos called these rings $H$-rings and showed that any commutative noetherian ring is Camillo (see [15, p. 110]).

Example 3.5. (i) The ring of continuous functions from $[0,1]$ to itself is a Camillo ring (see [6, p. 1461]).

(ii) By [10, Theorem 6.1A], every ring of Krull dimension zero is a Camillo ring.

(iii) Using [19, Lemma 2.3 and Propositions 3.2 and 4.1], we conclude that if $R$ is a ring such that $R_{\mathfrak{m}}$ is a noetherian ring for every maximal ideal $\mathfrak{m}$ of $R$, then $R$ is a Camillo ring. In particular, any von Neumann regular ring is Camillo.

Remark 3.6. It is shown in [21, Satz 2.5] that over a Camillo ring $R$, any supplemented $R$-module is locally supplemented.

A module $M$ is called subdirectly irreducible if $M$ has a simple essential socle. It is easy to see that an $R$-module $M$ is subdirectly irreducible if and only if 
$E(M)=E(S)$ for some simple submodule $S$ of $M$. Recall that a module $M$ is called finitely embedded if there exist finitely many simple submodules $S_{i}(1 \leq i \leq n)$ of $M$ such that $E(M)=E\left(S_{1}\right) \oplus \cdots \oplus E\left(S_{n}\right)$. Next, we characterize the class of rings $R$ for which every finitely embedded $R$-module is locally supplemented. It turns out that this class is exactly that of the Camillo rings.

Theorem 3.7. Let $R$ be a ring. The following conditions are equivalent:

(i) $R$ is a Camillo ring;

(ii) $E(R / \mathfrak{m})$ is locally supplemented for every $\mathfrak{m} \in \operatorname{Max}(R)$;

(iii) $\bigoplus_{\mathfrak{m} \in \operatorname{Max}(R)} E(R / \mathfrak{m})$ is a locally supplemented $R$-module;

(iv) Any subdirectly irreducible R-module is locally supplemented;

(v) Any finitely embedded R-module is locally supplemented;

(vi) $\operatorname{Max} \operatorname{Supp}(E(S))=\operatorname{Max} \operatorname{Supp}(S)$ for any simple $R$-module $S$;

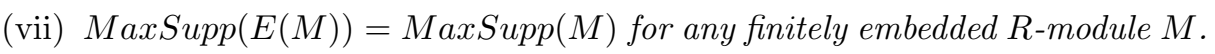

Proof. Note that the class of locally supplemented modules is closed under submodules and direct sums (see Remark 2.8).

(i) $\Leftrightarrow$ (ii) This follows from Proposition 2.12.

(ii) $\Rightarrow$ (iii) This is clear.

(iii) $\Rightarrow$ (iv) Let $M$ be a subdirectly irreducible $R$-module. So there exists a maximal ideal $\mathfrak{m}$ of $R$ such that $E(M) \cong E(R / \mathfrak{m})$. By hypothesis, $E(M)$ is a locally supplemented $R$-module. This implies that $M$ is locally supplemented.

(iv) $\Rightarrow(\mathrm{v})$ Let $M$ be a finitely embedded $R$-module. Then $E(M)$ is a finite direct sum of subdirectly irreducible $R$-modules. By (iv), $E(M)$ is locally supplemented. So $M$ itself is a locally supplemented module.

(v) $\Rightarrow$ (vi) Let $S$ be a simple $R$-module. As $E(S)$ is finitely embedded, $E(S)$ is locally supplemented. By Proposition 2.12, there exists a maximal ideal $\mathfrak{m}$ of $R$ such that $\operatorname{MaxSupp}(E(S))=\{\mathfrak{m}\}$. This clearly forces $\operatorname{MaxSupp}(S)=\{\mathfrak{m}\}$.

(vi) $\Rightarrow$ (vii) Let $M$ be a finitely embedded $R$-module. Then $E(M)=E\left(S_{1}\right) \oplus \cdots \oplus$ $E\left(S_{n}\right)$ for some simple submodules $S_{i}(1 \leq i \leq n)$ of $M$. Thus MaxSupp $(E(M))=$ $\cup_{i=1}^{n} \operatorname{Max} \operatorname{Supp}\left(E\left(S_{i}\right)\right)$ (see [4, p. 133 Proposition 16]). By hypothesis, we have $\operatorname{Max} \operatorname{Supp}(E(M))=\cup_{i=1}^{n} \operatorname{Max} \operatorname{Supp}\left(S_{i}\right) \subseteq \operatorname{MaxSupp}(M)$. Hence $\operatorname{Max} \operatorname{Supp}(M)=$ $\operatorname{Max} \operatorname{Supp}(E(M))$.

(vii) $\Rightarrow$ (ii) Let $\mathfrak{m} \in \operatorname{Max}(R)$. Since $E(R / \mathfrak{m})$ is a finitely embedded $R$-module, $\operatorname{Max} \operatorname{Supp}(E(R / \mathfrak{m}))=\operatorname{MaxSupp}(R / \mathfrak{m})=\{\mathfrak{m}\}$. Applying Proposition 2.12, it follows that $E(R / \mathfrak{m})$ is a locally supplemented $R$-module. 
Example 3.8. Combining Proposition 3.1 and Theorem 3.7, we see that any semiperfect ring is Camillo.

Let $M$ be an $R$-module. We say that $M$ is an LE-module if $\operatorname{End}_{R}(M)$ is a local ring. It is well known that every LE-module is indecomposable. A decomposition $M=\oplus_{i \in I} M_{i}$ is called an LE-decomposition of $M$ if each $M_{i}(i \in I)$ is an LEmodule. Let $\operatorname{Ass}_{f}(M)$ be the set of weakly associated primes of $M$, that is, the set of prime ideals $\mathfrak{p}$ of $R$ such that $\mathfrak{p}$ is minimal among the prime ideals containing $A n n_{R}(x)$ for some $x \in M$. Recall that a ring $R$ is called a Gelfand ring if every prime ideal of $R$ is contained in only one maximal ideal of $R$. A nonzero module $M$ is said to have finite uniform (or Goldie) dimension if there exist a positive integer $n$ and an essential submodule $V$ of $M$ such that $V$ is a direct sum of $n$ uniform submodules. In this case, we write $u \operatorname{dim}(M)=n$. If $M=0$ we set $u \cdot \operatorname{dim}(M)=0$.

Replacing "m $\in \operatorname{Max}(R)$ " in the condition (ii) of Theorem 3.7 by "p $\in \operatorname{Spec}(R)$ ", we obtain the following characterizations of Gelfand rings in terms of locally supplemented modules.

Theorem 3.9. The following conditions are equivalent for a ring $R$ :

(i) $R$ is a Gelfand ring;

(ii) For every prime ideal $\mathfrak{p}$ of $R, E(R / \mathfrak{p})$ is locally supplemented;

(iii) $\bigoplus_{\mathfrak{p} \in \operatorname{Spec}(R)} E(R / \mathfrak{p})$ is a locally supplemented $R$-module;

(iv) Any LE-R-module is locally supplemented;

(v) Any R-module having an LE-decomposition is locally supplemented;

(vi) Any $R$-module $M$ such that $E(M)$ has an indecomposable decomposition is a locally supplemented module;

(vii) Any $R$-module $M$ which has a semiperfect endomorphism ring is a locally supplemented module;

(viii) Any R-module having a finite Goldie dimension is locally supplemented;

(ix) Any R-module $M$ such that $A_{s} s_{f}(M)$ is finite is locally supplemented.

Proof. (i) $\Rightarrow$ (iv) By [8, Proposition III.1], MaxSupp $(M)$ contains only one maximal ideal $\mathfrak{m}$ of $R$. Thus $M=K_{\mathfrak{m}}(M)$. Therefore $M$ is locally supplemented by Corollary 2.10 .

(iv) $\Rightarrow$ (ii) This follows from [15, Lemma 2.29 and Proposition 3.12].

(ii) $\Rightarrow$ (i) Let $\mathfrak{p}$ be a prime ideal of $R$. Note that any maximal ideal of $R$ containing $\mathfrak{p}$ belongs to $\operatorname{MaxSupp}(E(R / \mathfrak{p}))$. But $\operatorname{MaxSupp}(E(R / \mathfrak{p}))$ contains only one element by Proposition 2.12. Then $\mathfrak{p}$ is contained in only one maximal ideal of $R$. It follows that $R$ is a Gelfand ring. 
(ii) $\Leftrightarrow$ (iii) and (iv) $\Rightarrow$ (v) See Remark 2.8.

(v) $\Rightarrow$ (vii) This follows from the fact that any module $M$ with a semiperfect endomorphism ring has an LE-decomposition (see [9, Proposition 3.14]).

(vii) $\Rightarrow$ (iv) This is immediate.

(v) $\Rightarrow$ (vi) Note that every indecomposable injective module has a local endomorphism ring by [15, Proposition 3.12]. This implication follows directly from Remark 2.8(i) and the fact that $M \leq E(M)$ for any module $M$.

(vi) $\Rightarrow$ (viii) Let $M$ be an $R$-module having a finite Goldie dimension. By [12, Theorem 13.3], $E(M)$ is a finite direct sum of indecomposable injective modules. The result follows from (vi).

(viii) $\Rightarrow$ (i) Let $\mathfrak{p}$ be a prime ideal of $R$. Then $R / \mathfrak{p}$ is a uniform $R$-module. So the $R$-module $R / \mathfrak{p}$ is locally supplemented. Since $R / \mathfrak{p}$ is an indecomposable $R$-module, $\operatorname{Max} \operatorname{Supp}(R / \mathfrak{p})=\{\mathfrak{m}\}$ for some $\mathfrak{m} \in \operatorname{Max}(R)$ by Corollary 2.10. Therefore $\mathfrak{m}$ is the only maximal ideal of $R$ that contains $\mathfrak{p}$. Thus $R$ is a Gelfand ring.

(ix) $\Rightarrow$ (i) Let $\mathfrak{p}$ be a prime ideal of $R$. We have $A s s_{f}(R / \mathfrak{p})=\{\mathfrak{p}\}$ is finite and so $R / \mathfrak{p}$ is locally supplemented. By similar arguments as in the proof of the implication (viii) $\Rightarrow$ (i), we obtain that $R$ is a Gelfand ring.

(i) $\Rightarrow$ (ix) Let $M$ be an $R$-module such that $A s s_{f}(M)$ is finite and let $0 \neq x \in$ $M$. Since $A s s_{f}(R x) \subseteq A s s_{f}(M), A s s_{f}(R x)$ is finite. This implies that the ring $R / \operatorname{Ann}(x)$ has a finite number of minimal prime ideals. But $R / A n n(x)$ is also Gelfand. So $R / \operatorname{Ann}(x)$ is semiperfect by [1, Theorem 5]. Therefore $M$ is locally supplemented by Proposition 2.6. This completes the proof.

To obtain another characterization of Gelfand rings, we need the following lemma.

Lemma 3.10. Let $R$ be a ring and let $M$ be an R-module. Then $M$ is a locally supplemented $R$-module if and only if $M \otimes N$ is a locally supplemented $R$-module for every $R$-module $N$.

Proof. $(\Rightarrow)$ Let $N$ be an $R$-module and let $0 \neq x=\sum_{i=1}^{n} x_{i} \otimes y_{i} \in M \otimes N$ where $x_{i} \in M$ and $y_{i} \in N$ for every $i \in\{1, \ldots, n\}$. Let $M^{\prime}=R x_{1}+\cdots+$ $R x_{n}$. Thus $\operatorname{Ann}\left(M^{\prime}\right) \subseteq \operatorname{Ann}(R x)$. Note that $M^{\prime}$ is locally supplemented as $M^{\prime} \leq$ $M$. Then $R / A n n\left(M^{\prime}\right)$ is a semiperfect ring by Lemma 2.5. Since $R / A n n(R x) \cong$ $\frac{R / A n n\left(M^{\prime}\right)}{A n n(R x) / A n n\left(M^{\prime}\right)}$ (as rings), it follows that $R / A n n(x)$ is also a semiperfect ring. Therefore $M \otimes N$ is locally supplemented by Proposition 2.6 .

$(\Leftarrow)$ Take $N=R$.

Let $M$ be an $R$-module and let $\mathfrak{p}$ be a prime ideal of $R$. Since the ring $R_{\mathfrak{p}}$ is local (and hence semiperfect), $M_{\mathfrak{p}}$ is always a locally supplemented $R_{\mathfrak{p}}$-module 
(Proposition 3.1). But this is no longer true if we consider $M_{\mathfrak{p}}$ as an $R$-module as shows the following result.

Corollary 3.11. The following are equivalent for a ring $R$ :

(i) For every prime ideal $\mathfrak{p}$ of $R, R_{\mathfrak{p}}$ is a locally supplemented $R$-module;

(ii) For every prime ideal $\mathfrak{p}$ of $R$ and for every $R$-module $M, M_{\mathfrak{p}}$ is a locally supplemented $R$-module;

(iii) $\oplus_{\mathfrak{p} \in \operatorname{Spec}(R)} R_{\mathfrak{p}}$ is a locally supplemented $R$-module;

(iv) $\oplus_{\mathfrak{p} \in \operatorname{Spec}(R)} M_{\mathfrak{p}}$ is a locally supplemented $R$-module for every $R$-module $M$;

(v) $R$ is Gelfand.

Proof. (i) $\Rightarrow$ (ii) It is well known that for every prime ideal $\mathfrak{p}$ of a ring $R$ and for every $R$-module $M, M_{\mathfrak{p}} \cong M \otimes R_{\mathfrak{p}}$. The result now follows from Lemma 3.10.

The implication (ii) $\Rightarrow$ (i) is clear.

(i) $\Leftrightarrow$ (iii) and (ii) $\Leftrightarrow$ (iv) See Remark 2.8 .

(i) $\Rightarrow$ (v) Let $\mathfrak{p}$ be a prime ideal of $R$. Then $R_{\mathfrak{p}}$ is an indecomposable $R$-module. Since $R_{\mathfrak{p}}$ is a locally supplemented $R$-module, $\operatorname{Max} \operatorname{Supp}\left(R_{\mathfrak{p}}\right)$ contains only one element by Corollary 2.10. Moreover, if $\mathfrak{m}$ is a maximal ideal containing $\mathfrak{p}$, then $\mathfrak{m} \in \operatorname{Max} \operatorname{Supp}\left(R_{\mathfrak{p}}\right)$. So $\mathfrak{p}$ is contained in only one maximal ideal of $R$. Therefore $R$ is a Gelfand ring.

(v) $\Rightarrow$ (i) Let $\mathfrak{p}$ be a prime ideal of $R$. Then $R_{\mathfrak{p}} \cong \operatorname{End}_{R}\left(R_{\mathfrak{p}}\right)$ is a local ring. Now apply Theorem 3.9.

Corollary 3.11 should be contrasted with the following remark.

Remark 3.12. Let $R$ be a ring. Note that the $R$-module $\prod_{\mathfrak{p} \in \operatorname{Spec}(R)} R_{\mathfrak{p}}$ is locally supplemented if and only if $R$ is a semiperfect ring. This comes from the fact that $R$ is isomorphic (as an $R$-module) to a submodule of $\prod_{\mathfrak{p} \in \operatorname{Spec}(R)} R_{\mathfrak{p}}$ (see Proposition $3.1)$.

A ring $R$ is called clean if each element of $R$ can be expressed as the sum of a unit and an idempotent. By [1, Proposition 2], every local ring is clean. It is shown in [1, Corollary 4] that any clean ring is Gelfand. Combining Theorems 3.7 and 3.9 , we get the following hierarchy:

$$
R \text { is clean } \Rightarrow R \text { is Gelfand } \Rightarrow R \text { is Camillo. }
$$

It is well known that for any prime ideal $\mathfrak{p}$ of $R$, the $R$-module $E(R / \mathfrak{p})$ is indecomposable. Replacing the condition " $E(R / \mathfrak{p})$ is locally supplemented for all $\mathfrak{p} \in \operatorname{Spec}(R)$ " in Theorem 3.9 by the condition "any indecomposable $R$-module is locally supplemented", we get the following characterization of clean rings. 
Proposition 3.13. The following conditions are equivalent for a ring $R$ :

(i) $R$ is a clean ring;

(ii) Any indecomposable $R$-module is locally supplemented;

(iii) Any R-module having an indecomposable decomposition is locally supplemented.

Proof. (i) $\Leftrightarrow$ (ii) By [8, Proposition III.2].

(ii) $\Leftrightarrow$ (iii) This follows from Remark 2.8.

Let $M$ be an $R$-module. We say that $M$ is $\sum$-injective if any direct sum of copies of $M$ is injective. Clearly, any $\sum$-injective $R$-module is injective and the converse holds when $R$ is a noetherian ring.

Proposition 3.14. Let $R$ be a ring. Then the following conditions are equivalent:

(i) Any $\sum$-injective $R$-module is locally supplemented;

(ii) For any prime ideal $\mathfrak{p}$ of $R$ such that $R_{\mathfrak{p}}$ is a noetherian ring, $E(R / \mathfrak{p})$ is a locally supplemented $R$-module.

Proof. (i) $\Rightarrow$ (ii) Let $\mathfrak{p} \in \operatorname{Spec}(R)$ such that $R_{\mathfrak{p}}$ is a noetherian ring. By [3, Remark p. 236], $E(R / \mathfrak{p})$ is $\sum$-injective and so it is locally supplemented.

(ii) $\Rightarrow$ (i) Let $M$ be a $\sum$-injective $R$-module. By [3, Theorem 1.9], there exists $H \subseteq \operatorname{Spec}(R)$ such that $M \cong \oplus_{\mathfrak{p} \in H} E(R / \mathfrak{p})$ and $E(R / \mathfrak{p})$ is $\sum$-injective for every $\mathfrak{p} \in H$. Using again [3, Remark p. 236], it follows that each $R_{\mathfrak{p}}(\mathfrak{p} \in H)$ is a noetherian ring. Hence each $E(R / \mathfrak{p})(\mathfrak{p} \in H)$ is a locally supplemented $R$-module. Therefore $M$ is locally supplemented (see Remark 2.8 ).

Corollary 3.15. Let $R$ be a Gelfand ring. Then any $\sum$-injective $R$-module is locally supplemented.

Proof. By Theorem 3.9 and Proposition 3.14.

We conclude this paper by investigating the class of rings $R$ for which every locally supplemented $R$-module is semisimple. We call a ring $R$ an $L S$-ring if every local $R$-module is a simple module. It is clear that every von Neumann regular ring is an LS-ring

Proposition 3.16. The following statements are equivalent for a ring $R$ :

(i) Any locally supplemented R-module is semisimple;

(ii) For any $R$-module $M, K(M)=\operatorname{Soc}(M)$;

(iii) For any locally supplemented $R$-module $M, \operatorname{Rad}(M)=0$;

(iv) $R$ is an LS-ring. 
Proof. (i) $\Leftrightarrow$ (ii) This equivalence is immediate.

(i) $\Rightarrow$ (iii) $\Rightarrow$ (iv) These implications are clear.

(iv) $\Rightarrow$ (i) Let $M$ be a locally supplemented $R$-module. By Theorem 2.9, $M=$ $\bigoplus_{\mathfrak{m} \in \operatorname{MaxSupp}(M)} K_{\mathfrak{m}}(M)$. Fix $\mathfrak{m} \in \operatorname{MaxSupp}(M)$ and let $0 \neq x \in K_{\mathfrak{m}}(M)$. Then $R / A n n(x)$ is a local ring. This implies that $R / A n n(x)$ is a local $R$-module. By hypothesis, $R / A n n(x)$ is a simple $R$-module. Therefore $R / A n n(x) \cong R x$ is a simple $R$-module. Thus $K_{\mathfrak{m}}(M)$ is semisimple for all $\mathfrak{m} \in \operatorname{Max} \operatorname{Supp}(M)$. It follows that $M$ is a semisimple $R$-module.

In the next results, we shed some light on the structure of LS-rings.

Proposition 3.17. Let $R$ be a ring with $J=\operatorname{Rad}(R)$. Then the following conditions are equivalent:

(i) $R$ is an $L S$-ring;

(ii) (1) $R / J$ is an LS-ring, and

(2) For any maximal ideal $\mathfrak{m}$ of $R, \operatorname{Rad}(\mathfrak{m}) \ll \mathfrak{m}$, and

(3) $\mathfrak{m}^{2}=\mathfrak{m}$ for any maximal ideal $\mathfrak{m}$ of $R$;

(iii) (1) $R / J$ is an LS-ring, and

(2) $J \ll \mathfrak{m}$ for any maximal ideal $\mathfrak{m}$ of $R$.

Proof. We first observe that for a maximal ideal $\mathfrak{m}$ of $R$ with $\mathfrak{m}^{2}=\mathfrak{m}$, we have $\operatorname{Rad}(\mathfrak{m})=\cap_{\mathfrak{m}^{\prime} \in \operatorname{Max}(R)} \mathfrak{m}^{\prime} \mathfrak{m}=J($ see [11, Lemma 3]).

(i) $\Rightarrow$ (ii) It is easily seen that $R$ satisfies the condition (1). To prove (3), let $\mathfrak{m}$ be a maximal ideal of $R$. Since $R / \mathrm{m}^{2}$ is a local $R$-module, $R / \mathrm{m}^{2}$ is a simple $R$-module. This yields $\mathfrak{m}^{2}=\mathfrak{m}$. Let us show that $R$ satisfies (2). Let $X$ be a submodule of $\mathfrak{m}$ such that $X+\operatorname{Rad}(\mathfrak{m})=\mathfrak{m}$. Since $\operatorname{Rad}(\mathfrak{m})=J$, we have $X+J=\mathfrak{m}$. This implies that $\mathfrak{m}$ is the only maximal ideal of $R$ which contains $X$. Therefore $R / X$ is a local $R$-module. By hypothesis, we have $X=\mathfrak{m}$. Hence $\operatorname{Rad}(\mathfrak{m}) \ll \mathfrak{m}$.

(ii) $\Rightarrow$ (iii) This is obvious.

(iii) $\Rightarrow$ (i) Let $\mathfrak{a}$ be an ideal of $R$ such that $\mathfrak{a}$ is contained in only one maximal ideal $\mathfrak{m}$ of $R$. Then $R /(\mathfrak{a}+J)$ is a local $R$-module. Thus $R /(\mathfrak{a}+J)$ is a local $R / J$-module. Since $R / J$ is an LS-ring, we have $\mathfrak{a}+J=\mathfrak{m}$. As $J \ll \mathfrak{m}$, we see that $\mathfrak{a}=\mathfrak{m}$. This completes the proof.

Next, we provide a characterization of when a Camillo ring is an LS-ring.

Proposition 3.18. Let $R$ be a Camillo ring. Then the following conditions are equivalent:

(i) $R$ is an LS-ring; 
(ii) $R$ is von Neumann regular.

Proof. (i) $\Rightarrow$ (ii) Let $\mathfrak{m}$ be a maximal ideal of $R$. Since $R$ is Camillo, $E(R / \mathfrak{m})$ is a locally supplemented $R$-module by Theorem 3.7. Therefore $E(R / \mathfrak{m})$ is a semisimple $R$-module by Proposition 3.16. Thus $R / \mathfrak{m}=E(R / \mathfrak{m})$ is an injective $R$-module. So all simple $R$-modules are injective. By [12, Corollary 3.73], $R$ is a von Neumann regular ring.

(ii) $\Rightarrow$ (i) This is clear.

Recall that a module $M$ is called semiartinian is any nonzero factor module of $M$ contains a simple submodule. We end this article by characterizing Camillo rings over which any locally supplemented module can be decomposed into a direct sum of cyclic submodules.

Theorem 3.19. The following conditions are equivalent for a Camillo ring $R$ :

(i) Any locally supplemented R-module is a direct sum of cyclic submodules;

(ii) $R_{\mathfrak{m}}$ is an artinian principal ideal ring for every maximal ideal $\mathfrak{m}$ of $R$.

Proof. (i) $\Rightarrow$ (ii) By [2, Proposition 8.8], we only need to show that $R_{\mathfrak{m}}$ is an artinian ring and $\operatorname{dim}_{R_{\mathfrak{m}} / \mathfrak{m} R_{\mathfrak{m}}}\left(\mathfrak{m} R_{\mathfrak{m}} / \mathfrak{m}^{2} R_{\mathfrak{m}}\right) \leq 1$ for every maximal ideal $\mathfrak{m}$ of $R$.

Let $\mathfrak{m}$ be a maximal ideal of $R$ and let $M$ be a noetherian semiartinian $R$ module. By [16, Proposition 3.1], we see that $M$ is an artinian module. Thus $M$ is locally supplemented. By assumption, it follows that $M$ is a direct sum of cyclic submodules. Using [17, Lemma 1], we have $\operatorname{dim}_{R / \mathfrak{m}} \mathfrak{m} / \mathfrak{m}^{2} \leq 1$. This yields $\operatorname{dim}_{R_{\mathfrak{m}} / \mathfrak{m} R_{\mathfrak{m}}}\left(\mathfrak{m} R_{\mathfrak{m}} / \mathfrak{m}^{2} R_{\mathfrak{m}}\right) \leq 1$.

Now since $R$ is a Camillo ring, it follows that any finitely embedded $R$-module is locally supplemented by Theorem 3.7. Therefore every finitely embedded $R$-module is a finite direct sum of cyclic submodules. Applying [18, Theorem 3], we conclude that $R_{\mathfrak{m}}$ is an artinian ring for every maximal ideal $\mathfrak{m}$ of $R$.

(ii) $\Rightarrow$ (i) let $M$ be a locally supplemented $R$-module. By Theorem 2.9, $M=$ $\bigoplus_{\mathfrak{m} \in \operatorname{Max} \operatorname{Supp}(M)} K_{\mathfrak{m}}(M)$. Let $\mathfrak{m} \in \operatorname{Max} \operatorname{Supp}(M)$. Notice that $K_{\mathfrak{m}}(M)$ has also a structure of an $R_{\mathfrak{m}}$-module defined by the following operation: given $x \in K_{\mathfrak{m}}(M)$, $r \in R$ and $s \in R \backslash \mathfrak{m}$, we put $(r / s) x=r x^{\prime}$ with $x^{\prime}$ is the unique element of $K_{\mathfrak{m}}(M)$ which satisfies $x=s x^{\prime}$. Note that $x^{\prime}$ exists because $A n n(x)+R s=R$. Moreover, we have $R_{\mathfrak{m}} x=R x$ for every $x \in K_{\mathfrak{m}}(M)$. By hypothesis, $R_{\mathfrak{m}}$ is an artinian principal ideal ring. So $K_{\mathfrak{m}}(M)$ is a direct sum of cyclic $R_{\mathfrak{m}}$-submodules by $[15$, Theorem $6.7]$. Hence $M$ is a direct sum of cyclic $R$-submodules. 


\section{References}

[1] D. D. Anderson and V. P. Camillo, Commutative rings whose elements are a sum of a unit and idempotent, Comm. Algebra, 30(7) (2002), 3327-3336.

[2] M. F. Atiyah and I. G. Macdonald, Introduction to Commutative Algebra, Addison-Wesley Publishing Co., Reading, Mass.-London-Don Mills, Ont., 1969.

[3] I. Beck, $\Sigma$-injective modules, J. Algebra, 21(2) (1972), 232-249.

[4] N. Bourbaki, Éléments de Mathématique, Algèbre Commutative, Chapitres 1 et 2, Masson, Paris, 1985.

[5] E. Büyükaşı and C. Lomp, Rings whose modules are weakly supplemented are perfect. Applications to certain ring extensions, Math. Scand., 105(1) (2009), 25-30.

[6] V. Camillo, Homological independence of injective hulls of simple modules over commutative rings, Comm. Algebra, 6(14) (1978), 1459-1469.

[7] J. Clark, C. Lomp, N. Vanaja and R. Wisbauer, Lifting Modules, Supplements and Projectivity in Module Theory, Frontiers in Mathematics, Birkhäuser Verlag, Basel, 2006.

[8] F. Couchot, Indecomposable modules and Gelfand rings, Comm. Algebra, 35(1) (2007), 231-241.

[9] A. Facchini, Module Theory, Endomorphism rings and direct sum decompositions in some classes of modules, Progress in Mathematics, 167, Birkhäuser Verlag, Basel, 1998.

[10] C. Faith, Minimal cogenerators over Osofsky and Camillo rings, Advances in Ring Theory (Granville, OH, 1996), Trends Math., Birkhäuser Boston, Boston, MA, (1997), 105-118.

[11] A. I. Generalov, w-cohigh purity in a category of modules, Mat. Zametki, 33(5) (1983), 402-408; translation from Mat. Zametki, 33(5) (1983), 785-796.

[12] T. Y. Lam, Lectures on Modules and Rings, Graduate Texts in Mathematics, 189, Springer-Verlag, New York, 1999.

[13] S. McAdam, Deep decompositions of modules, Comm. Algebra, 26(12) (1998), 3953-3967.

[14] S. H. Mohamed and B. J. Müller, Continuous and Discrete Modules, London Mathematical Society Lecture Note Series, 147, Cambridge University Press, Cambridge, 1990. 
[15] D. W. Sharpe and P. Vámos, Injective Modules, Cambridge Tracts in Mathematics and Mathematical Physics, No. 62, Cambridge University Press, London-New York, 1972.

[16] R. C. Shock, Dual generalizations of the artinian and noetherian conditions, Pacific J. Math., 54(2) (1974), 227-235.

[17] T. S. Shores, Decompositions of finitely generated modules, Proc. Amer. Math. Soc., 30(3) (1971), 445-450.

[18] P. Vámos, The dual of the notion of "finitely generated", J. London Math. Soc., 43(1) (1968), 643-646.

[19] P. Vámos, Classical rings, J. Algebra, 34(1) (1975), 114-129.

[20] H. Zöschinger, Komplementierte Moduln über Dedekindringen, J. Algebra, 29(1) (1974), 42-56.

[21] H. Zöschinger, Gelfandringe und koabgeschlossene Untermoduln, Bayer. Akad. Wiss. Math.-Natur. KI. Sitzungsber., 3 (1982), 43-70.

\section{Farid Kourki}

Centre Régional des Métiers de l'Education et de la Formation (CRMEF-TTH)

Annexe de Larache, B.P. 4063

Larache, Morocco

e-mail: kourkifarid@hotmail.com

Rachid Tribak (Corresponding Author)

Centre Régional des Métiers de l'Education et de la Formation (CRMEF-TTH)-Tanger

Avenue My Abdelaziz, Souani, B.P. 3117

Tangier, Morocco

e-mail: tribak12@yahoo.com 